\title{
NeuroSearch's dopaminergic stabilizer improves movement disorders in Huntington's disease
}

\begin{abstract}
A Phase III trial showed that the dopaminemodulating agent pridopidine met the primary end point of improving voluntary motor function in patients with Huntington's disease, as well as improving some involuntary motor symptoms associated with the disorder. In the trial - known as MermaiHD - 437 patients were treated with $45 \mathrm{mg}$ of pridopidine either once- or twice-daily for 26 weeks, with the treatment effect in the twice-daily cohort reaching statistical significance.

"These results are exciting, as pridopidine targets some movement disorders such as dystonia (writhing movements) that have previously not been possible to treat," says Michael Hayden, Professor of Medical Genetics and Director of the Centre for Molecular Medicine and Therapeutics at the University of British Columbia, Canada. "Huntington's disease represents a tremendous unmet medical need," he adds.

The drug targets the abnormalities in dopaminergic function that are associated with Huntington's disease, which contribute to the motor movement disorders that are characteristic of the condition. Pridopidine is a so-called dopaminergic stabilizer
\end{abstract}

that has novel pharmacological properties U. Med Chem. 15 Feb 2010; doi:10.1021/ jm901689v). It is an antagonist of the $D_{2}$ dopamine receptor, a $\mathrm{G}$ protein-coupled receptor (GPCR) found in striatal brain regions. Preclinical studies indicate that - in contrast to conventional GPCR antagonists - pridopidine preferentially binds to receptors that are in the 'active' state; that is, those that are coupled to the $G$ protein. In addition, it has rapid receptor-dissociation kinetics. These properties enable it to display state-dependent antagonism at the $D_{2}$ receptor: increasing dopaminergic activity when dopamine levels are too low, reducing activity when dopamine levels are too high, and without effect when the transmitter is at physiological levels.

Although pridopidine reduced dystonia, it did not affect all types of movement disorders that are associated with the condition. "It is important to note that the drug had no effect on chorea (quick movements), which is the most prominent movement disorder associated with Huntington's disease," highlights Hayden. Chorea can be ameliorated by treatment with tetrabenazine (Xenazine; Lundbeck), an inhibitor of vesicular monoamine transporter 2 and the only approved agent for Huntington's disease (Nature Rev. Drug Discov. 8, 17-18; 2009). However, its use is associated with serious side effects such as depression and an increased risk of suicide. It is not known whether it is possible to combine pridopidine with tetrabenazine. Other drugs in clinical trials include latrepirdine from Pfizer and Medivation, which is in Phase III trials and thought to improve mitochondrial functions in conditions of cellular stress, and Novartis' AFQ056, an antagonist of metabotropic glutamate receptor 5 that is in Phase II trials.

As Hayden points out, pridopidine appears to be safe, but its effects might be short-lived. "Treatment decreased some of the movement disorders for a short periodthat is, months - but its longer-term effects are unknown. We would need further studies to know if the positive effects are going to be sustained."

According to NeuroSearch, the MermaiHD study is currently being followed up by a further 6-month open-label treatment period in patients who completed the 6-month randomized study. Results are expected later this year. 